\title{
Effect of Surface Deviation of Solid Insulation on Impulsive Flashover Voltages Under Varying Environmental Conditions
}

\author{
R.W. Macpherson, M. P. Wilson, I.V. Timoshkin, M. J. Given, S. J. MacGregor \\ Dept. Electronic \& Electrical Engineering, University of Strathclyde, 204 George Street, \\ Glasgow, G1 1XW, UK
}

\begin{abstract}
In pulsed power engineering, solid spacers are used to insulate high voltage parts from extraneous metal parts. The applied voltage at which a discharge process initiates is important in the design process. In this paper, a method to potentially increase the failure voltage under multiple environmental conditions, without increasing the length of the solid spacer, was investigated. Three insulating materials: Delrin (Polyoxymethylene), Ultem (Polyetherimide) and HDPE (High Density Polyethylene), were tested under a 100/700 ns impulse voltage. Cylindrical spacers made of these materials were located in the center of a parallel-plane electrode setup in air, which provided a quasi-uniform field distribution. Breakdown tests published in this paper were performed in a sealed container at air pressures of $-0.5,0$ and 0.5 bar gauge, with a constant relative humidity level of $<\mathbf{1 0 \%}$ RH. The materials were tested under both, negative and positive, polarity impulses. The surfaces of a set of solid spacers were subjected to a 'knurled' finish, where slight indentations are added to the surface of the materials, prior to testing, to allow comparison with the breakdown voltages for samples with 'smooth' (machined) surface finishes. For negative polarity impulses, the flashover voltage for smooth machined surfaces was generally higher than for knurled finishes. Under positive polarity impulses, the opposite effect was found to occur, where the flashover voltage of the samples with knurled surfaces was higher for all tests. The results will give designers, particularly within the pulsed power industry, information on flashover voltages of materials under an array of environmental conditions.
\end{abstract}

\section{INTRODUCTION}

Within pulsed power systems, the governing factor determining the overall breakdown strength of the system is often the voltage that initiates flashover, in the vicinity of solid insulating parts required to provide mechanical support. In this work, the application of deviations to the surfaces of solid spacer materials in dry air was investigated, in order to quantify the effect that the modified surface finish has on the flashover voltage of the insulation system.

Previous work on roughened surfaces has been conducted in $\mathrm{N}_{2}$ [1]. As the insulator surface roughness was increased from $0.1 \mu \mathrm{m}$ to $0.7 \mu \mathrm{m}$, the flashover voltage of the insulator first decreased, and then increased. This has been explained by absorption and a blocking effect of 'gullies' on the insulator surface. The positive polarity flashover voltages recorded were always lower than the negative polarity, irrespective of surface roughness. In [2], lightning impulse flashover voltage tests were conducted on samples of an epoxy resin, with varying surface roughness. A polarity effect was witnessed as the average surface roughness, $R_{a}$, was increased from $0.1 \mu \mathrm{m}$ to $1.4 \mu \mathrm{m}$, with the breakdown voltage under positive polarity increasing by $15 \mathrm{kV}$ beyond that recorded for negative polarity at $R_{a}=1.4 \mu \mathrm{m}$.

For surface deviations with larger dimensions, a review was completed in [3], wherein different shapes of insulating spacers have been proposed, rather than subjecting the surfaces of cylindrical spacers to a roughening process.

In this paper, a mixture of these tests will be completed, with cylindrical insulating spacers in the form of rods $40 \mathrm{~mm}$ in length and $30 \mathrm{~mm}$ in diameter, firstly on samples with a smooth machined surface for reference, as well as on samples with a novel 'knurled' surface finish. The knurled surface finish consists of diamond-shaped indentations, machined on to the surface of the materials. Experimental data has been generated on the flashover strength of samples of three solid materials, Delrin (Polyoxymethylene), HDPE (High Density Polyethylene) and Ultem (Polyetherimide). All tests were conducted at a relative humidity $(\mathrm{RH})$ of $<10 \%$, and at pressures of $-0.5,0$ and 0.5 bar gauge. Samples were subjected to negative and positive polarity impulsive voltages from a 10-stage Marx generator, with a 100/700 ns wave shape. The relative permittivity of each of the materials is $\varepsilon_{\mathrm{r}}=$ 2.3 for HDPE, $\varepsilon_{\mathrm{r}}=3.0$ for Ultem, and $\varepsilon_{\mathrm{r}}=3.8$ for Delrin. The average flashover initiation voltages from the resulting waveforms were determined and the standard deviation was calculated from the data set of 20 breakdown events, for each test. Thus, the flashover strength of samples of the three different materials could be compared for different surface finishes, different pressures, and for both, negative and positive, polarity.

\section{Methodology}

\section{A. Experimental Arrangement}

This section informs on the systems and methodology associated with practical testing. In Fig. 1a, the electrodespacer setup has been illustrated. This setup results in a quasiuniform electric field being produced during energisation, with an increase in the field intensity of $\sim 87 \%$ at the rounded edges of the HV electrode shown in Fig. 1b. These electrodes were connected to positive or negative $\mathrm{HV}$ and ground, respectively. A gas input was added in order to change the pressure of the air within the chamber during testing. 
a)

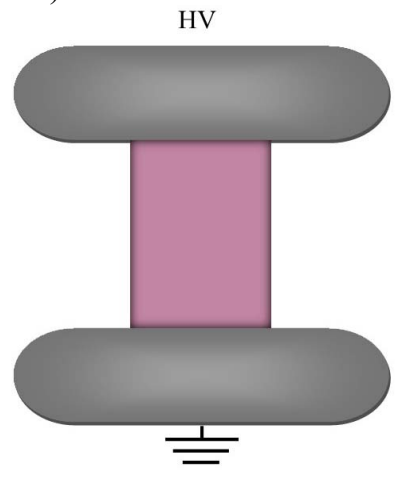

b)

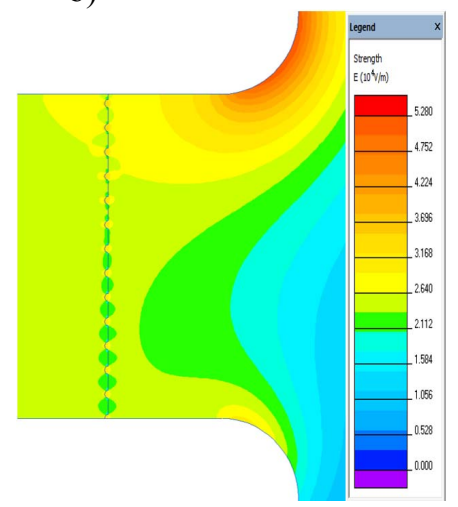

Fig. 1. a) Parallel-plane electrode setup with added dielectric spacer and

b) Electrostatic simulation of knurled spacer (shown in Fig. 2b), showing asymmetric field distribution from grounded boundary conditions added from test-cell location within faraday-caged lab.

The three solid materials used within tested are Delrin (Polyoxymethylene), HDPE (High Density Polyethylene) and Ultem (Polyetherimide). The insulation system is designed for use nominally at atmospheric pressure. Tests were conducted at \pm 0.5 bar gauge of atmospheric pressure at $-0.5,0$ and 0.5 bar gauge, in order to quantify the effect of small pressure fluctuations, either side of atmospheric pressure.

The charging voltage was supplied from a $100 \mathrm{kV}, 2.5 \mathrm{~mA}$, Glassman high voltage DC power supply, connected through a $1 \mathrm{M} \Omega$ charging resistor. This was then connected to the capacitor bank of the Marx generator. The $\mathrm{CuSO}_{4}$ waveshaping resistors had values of $300 \Omega$ for the wave tail and 700 $\Omega$ for the output resistor. This specific resistor arrangement resulted in a 100/700 ns output voltage waveform. The output of the Marx generator was connected to the test cell and a voltage divider arrangement in parallel. A 1000:1 Tektronix P6015A, $75 \mathrm{MHz}$ bandwidth $\mathrm{HV}$ probe was connected to the output (tap-off point) of an 8:1 $\mathrm{CuSO}_{4}$ resistive voltage divider, and through to a Tektronix MDO3012, $200 \mathrm{MHz}$ bandwidth oscilloscope for analysis of the resulting waveforms.

The testing procedure implemented was a 'step up' method, which is included in the ASTM D3426-97 standard [4] with $300 \mathrm{~V}$ charging increments, monitored on a DMM, through a 1000:1 Testec HVP-40 HV probe. The process was conducted 20 times for each set of test conditions, in order to find the average flashover voltage of these 20 breakdown events. A standard deviation was also calculated for each dataset, as illustrated using error bars. The average flashover voltages and standard deviations for each test can be seen within Figs. 3-5.

\section{B. Surface Deviations}

The surface modifications are introduced in this section. As aforementioned, the cylindrical spacers are $40 \mathrm{~mm}$ in length and $30 \mathrm{~mm}$ in diameter. Two different types of sample were tested in this paper. First, samples of each material with a smooth machined surface finish were produced. a)

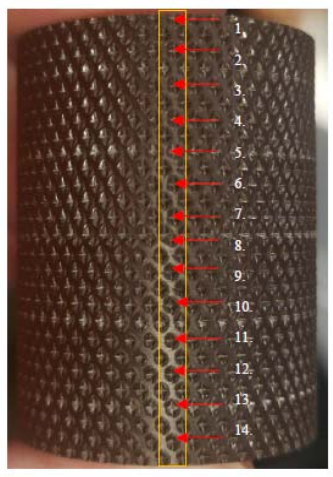

b)

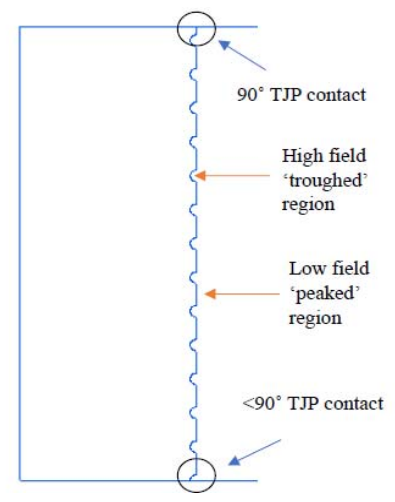

Fig. 2. Ultem knurled surface deviations in: a) Physical dielectric spacer; and b) Cross-sectional sketch of spacer surface; the yellow boxed area in a) shows the 14 indentations which are modelled in b), at a depth of $0.5 \mathrm{~mm}$; two different triple junction points were added in $b$ ) to show the potential dependence of the contact angle on the position of indentations

Also, surface-modified spacers were produced, with each spacer being subjected to a knurling process following manufacture. The end product is illustrated in Fig. 2a, with a cross-sectional sketch being illustrated in Fig. 2b. This process of knurling was chosen as it provides a very consistent and quick way of modifying a surface. The indentations applied to the surface of the spacers were $0.5 \mathrm{~mm}$ in depth, with $2.9 \mathrm{~mm}$ between each indentation.

\section{RESULTS}

\section{A. Graphical Results}

The generated experimental data is separated by pressure level here, enabling comparison of the performance of the three materials at different polarities and with different surface finishes. In each graph, data for an open-air gap, with no solid spacer, is also provided for comparison. Figs. 3, 4 and 5 show the graphical data for pressures of $-0.5,0$ and 0.5 bar gauge, respectively, showing the average flashover voltages and standard deviations (error bars).

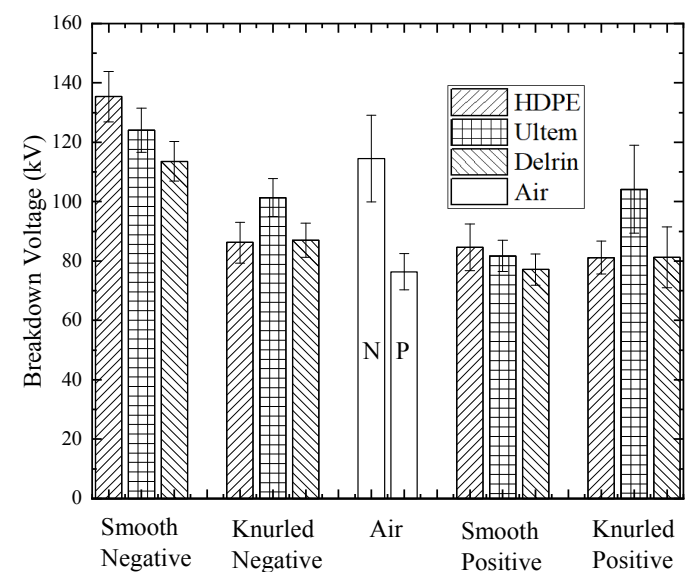

Fig. 3. Average flashover voltages and standard deviation for Delrin, Ultem and HDPE samples, with both smooth and knurled surface finishes, under negative and positive polarity, at a pressure of -0.5 bar gauge 


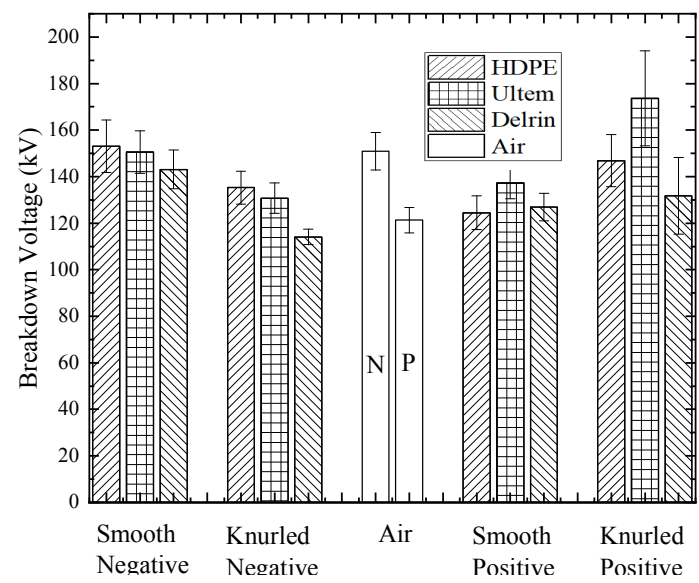

Fig. 4. Average flashover voltages and standard deviation for Delrin, Ultem and HDPE samples, with both smooth and knurled surface finishes, under negative and positive polarity, at a pressure of 0 bar gauge

In Fig. 3, the -0.5 bar gauge results are shown. Firstly, considering the results for open-air gaps, there is a substantial asymmetric performance for this nominally-symmetrical electrode setup, with the positive polarity voltage being $\sim 67 \%$ of the negative polarity voltage. For negative polarity, comparing the performance of the open-air gap to that of the gaps bridged with solid spacers, there are some differences in behaviour. The breakdown voltages with smooth machined surfaces are clearly higher than those with knurled surface finishes. It is also evident that the breakdown voltages are generally higher with the inclusion of a spacer (any material) with a smooth surface finish than for an open-air gap. The opposite behaviour is observed for samples with a knurled surface finish, the breakdown voltages being lower than that for the open-air gap, for all materials.

For positive polarity, there is a similar performance between the open-air gap, and for gaps bridged by samples with either a smooth or knurled surface finish, however there is a clear increase in the breakdown voltage for an Ultem spacer with a knurled surface finish, compared to all other positive polarity tests.

The results recorded at 0 bar gauge are shown in Fig. 4. Considering the results of the tests on open-air gaps, there is again a difference in the average breakdown voltage, with the positive polarity voltage being $\sim 80 \%$ of the negative polarity voltage. However, the difference in voltage magnitude is not as significant as that seen in Fig. 3. For negative polarity, again, the breakdown voltage of the gaps bridged by spacers with knurled finishes were less than those of gaps bridged by spacers with smooth machined finishes. However, spacers of all materials with smooth machined surfaces were seen to have very similar average breakdown voltage to that of the open-air gap.

For positive polarity, samples with a knurled surface finish generally had a higher breakdown voltage than those with a smooth machined surface finish, revealing an effect opposite to that observed for negative polarity. When compared to an open-air gap, gaps bridged by samples with smooth

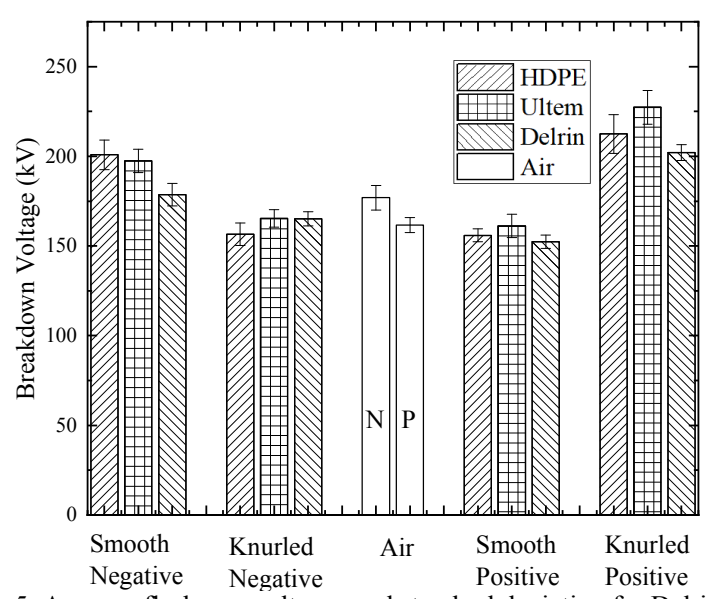

Fig. 5. Average flashover voltages and standard deviation for Delrin, Ultem and HDPE samples, with both smooth and knurled surface finishes, under negative and positive polarity, at a pressure of 0.5 bar gauge

machined surface finishes were seen to have very similar performance, for all materials.

The results gathered at 0.5 bar gauge are displayed in Fig. 5 . When looking at the difference in behaviour of the open-air gap with respect to polarity, an asymmetric performance is again evident, with the positive polarity voltage being $\sim 91 \%$ of the negative polarity voltage. This difference is not as significant as those evident in Figs. 3 and 4. This result shows that there is a correlation between the air pressure and the observed asymmetric breakdown performance of the parallelplane insulation system. For negative polarity impulses, the spacer surface finish influences the breakdown voltage of the gap. For spacers with smooth surface finish, the breakdown voltages are generally higher than that of the open-air gap, while the breakdown voltages for spacers with knurled surface finish are all slightly lower than that of the open-air gap.

For positive polarity impulses, the opposite behaviour is observed to that for negative polarity - for all materials, compared to the open-air gap, gaps bridged by spacers with a knurled surface finish have a higher breakdown voltage, while gaps bridged by spacers with a smooth surface finish have a similar or slightly lower breakdown voltage.

\section{B. Breakdown Visual Observations}

A photographic study was conducted using the electrodespacer setup shown in Fig. 1. This was done to facilitate potential discussion of the differences in breakdown voltage observed, in terms of the discharge location.

In this example, a HDPE spacer with a knurled surface was added between the electrodes, and 20 photographs (for 20 individual breakdown events) were taken at an applied voltage corresponding to the average flashover voltage from Fig. 5, at $217 \mathrm{kV}$ for positive polarity and $159 \mathrm{kV}$ for negative polarity. Displayed in Figs. 6a and 6b are example photographs showing the plasma channel location for each polarity. Two external mirrors have been added at different angles to the electrode-sample arrangement, to allow any discharge initiated behind the sample and out of the field of view of the camera to be captured. 
a)

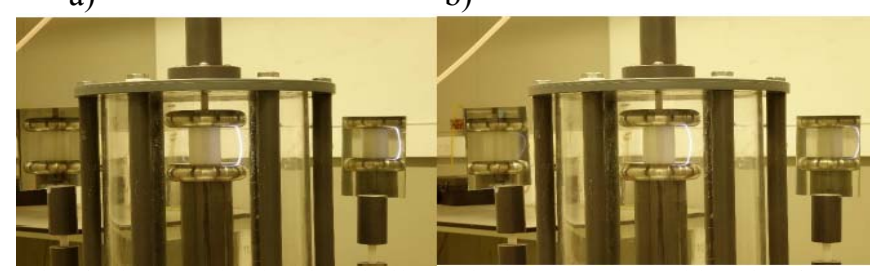

Fig. 6. Photographs showing similar typical plasma channel location for: a) Positive polarity; and b) Negative polarity

What can be seen from the two examples chosen is the very similar initiation and termination points for each polarity, with a brighter discharge for positive polarity due to the higher available energy, from the increased $50 \% V_{b r}$ compared to that for negative polarity. All open-shutter photographs captured during the study showed that, at $<10 \% \mathrm{RH}, 100 \%$ of the discharges were initiated and propagated across the air gap at the electrode edges, $\sim 10 \mathrm{~mm}$ from the material surface, for both polarities.

Viewing the experimental results reported here as a whole, although the discharges always occurred away from the surface of the solid spacers in this study (at $<10 \% \mathrm{RH}$ ), the effect of the inclusion of spacers with different characteristics on the breakdown voltage of the overall insulation system is evident.

\section{DISCUSSION}

In this study, breakdown channels were observed to consistently initiate and propagate at the outside edges of the electrodes, away from the surface of the solid sample, as shown by the photographic study summarised in Fig. 6 . Despite this, a polarity effect on the measured breakdown voltages is evident, with positive flashover voltages being higher than negative for gaps bridged by solid spacers with knurled surfaces, at pressures of 0 and 0.5 bar gauge. This result is particularly interesting since this behaviour opposes the polarity effect observed with no spacer, where the average negative breakdown voltage of the open-air gap is higher than that for positive polarity. The asymmetric field distribution in Fig. $1 \mathrm{~b}$ can account for this phenomenon, due to the grounding points within the lab.

This polarity effect in the case of spacers with knurled surfaces can potentially be explained by the different characteristics of positive and negative streamers. Both positive and negative streamers advance as a result of multiple electron avalanches in the streamer head. However, the exact mechanisms of streamer growth and propagation are different. Negative streamers advance due to a rapid outwards acceleration of the electrons constituting the streamer head, creating numerous electron avalanches directed away from the tip [5]. Positive streamers, on the other hand, propagate as a result of electron attraction to the positively-charged streamer head. For positive polarity, the electrons originate mainly from the region in front of the streamer, producing multiple avalanches directed towards the positive streamer head.

When using different surface modifications in relation to the different streamer mechanisms discussed, it should be considered that the spacer surface itself can be an efficient source of free electrons [6]. For knurled surfaces, the surface charge on the material can be mitigated, reducing the free electrons available through the added roughness to the material [7]. This corresponds with the empirical data found here, as positive streamers need free electrons some distance ahead in order to propagate to the point of breakdown [8], and the availability of free electrons is limited by the knurled surface; conversely, for samples with a smooth (machined) surface finish, this mitigation of free electrons on the surface of the solid dielectric is less probable, and breakdown ensues at lower applied voltages than for materials with a knurled surface finish. These physical mechanisms may explain the experimental results, particularly in Figs. 4 and 5.

\section{CONCLUSIONS AND FURTHER WORK}

It was found in the experimental study reported in this paper that negative breakdown voltages were higher for an open-air gap, and for gaps including smooth machined surfaces, than for gaps including knurled surfaces, on average, for all air pressures. Positive breakdown voltages were higher for gaps including knurled surfaces than for an open-air gap, or for gaps including smooth surfaces, on average, for all air pressures. For positive polarity, the benefit of knurling the sample surfaces is higher at 0.5 bar gauge compared to at the lower tested air pressures. In terms of the breakdown voltages recorded across all tests, the best performing material under negative polarity was HDPE (smooth surface finish), and under positive polarity was Ultem (knurled surface finish).

Further work will include repeating the breakdown voltage tests reported here, while increasing the level of relative humidity within the test chamber, to characterise changes in the behaviour of the average breakdown voltage for the different sets of test conditions.

\section{REFERENCES}

[1] P. Xue, S. Zhao, D. Xiao, L. Zhu and Y. Wu, "Experimental Research on Flashover Characteristics of Insulator with N2," 2018 IEEE Electrical Insulation Conference (EIC), San Antonio, TX, 2018, pp. 60-63.

[2] Yifan Qin et al., "A Study on Flashover Characteristics of Supporting Insulators in SF6 under Lightning Impulse," 2015 IEEE 11th International Conference on the Properties and Applications of Dielectric Materials (ICPADM). Page $604-607$

[3] A. H. Cookson, "Review of high-voltage gas breakdown and insulators in compressed gas," in IEE Proceedings A - Physical Science, Measurement and Instrumentation, Management and Education Reviews, vol. 128, no. 4, pp. 303-312, May 1981.

[4] ASTM D3426-97 Standard Test Method for Dielectric Breakdown Voltage and Dielectric Strength of Solid Electrical Insulating Materials Using Impulse Waves

[5] J. M. Meek and J. D. Craggs, Electrical Breakdown of Gases. London: Clarendon Press, 1953.

[6] Simulations and Measurements of Streamer discharges near Dielectrics Sigurd Gard Midttun MSc thesis.

[7] Effects of Roughening Insulator Surface on Charging and Flashover Characteristics of a Long Glass Insulator in Vacuum January 2006 IEEJ Transactions on Fundamentals and Materials 126(8):769-774

[8] Dubinova, A. A. (2016). Modeling of streamer discharges near dielectrics. Eindhoven: Technische Universiteit Eindhoven. 